INTERNATIONA

\title{
Research Article \\ BIODIVERSITY OF FUNGI FROM SILVER ORE WITH REFERENCE TO THE PRODUCTION OF SILVER NANOPARTICLES
}

Janakiraman Vardhana*, Govindarajan Kathiravan, Ravichandran Dhivya, Doss Vidhya

Department of Biotechnology, Vels University, Chennai, Tamil Nadu, India

*Corresponding Author Email: vardhana88@ymail.com

Article Received on: 01/12/14 Revised on: 28/12/14 Approved for publication: 06/01/15

\section{DOI: $10.7897 / 2230-8407.0617$}

\section{ABSTRACT}

Micro organisms take part in soil material transformation process and play an important role in soil formation, fertility evolution, plant nutrient in effect, soil structure formation and improvement and degradation and purification of toxic substances. As an important component of soil micro organism, fungi take a very important position in structure and function of ecosystem. The soil samples were collected and dilution technique and direct plating technique was used to determine the number of fungal CFU in soil samples and to isolate pure cultures. Fungi were identified according to their macroscopic and microscopic features. During the study the identified fungus are Aspergillus japonicus, Aspergillus tereus, Aspergillus nidulans, Aspergillus flavus, Bortyodiplodia, Aspergillus niger, Trichoderma, Monilia. The statistical report was done for the serial dilution techique to find out the maximum fungal growth and it was found to be Hypomycetes. As fungi thrive in competitive environments, it is hypothesized that their metabolic compatibility has been strongly influenced by natural selection. The most abundantly grown fungi were found to be Aspergillus niger and it is tested for the synthesis of silver nanoparticles.

Keywords: Silver ore soil, Hypomycetes, Aspergillus sp, Serial dilution, silver nanoparticles.

\section{INTRODUCTION}

Micro organisms take part in soil material transformation process and play an important role in soil formation, fertility evolution, plant nutrient in effect, soil structure formation and improvement and degradation and purification of toxic substances. ${ }^{1}$ As an important component of soil micro organism, fungi take a very important position in structure and function of ecosystem². 'True' fungi are ubiquitous in the environment and fulfill a range of important ecological functions, particularly those associated with nutrient and carbon cycling processes in soil. Fungi therefore have many different functions in soils, which includes both active roles, such as the degradation of dead plant material, or inactive roles where propagules are present in the soil as resting states. Current knowledge of fungal diversity in soil is based largely on observations of fruiting bodies present in an environment, or from cultures obtained from soil isolation exercises. Both of these approaches have serious limitations for the detection of the true diversity in any chosen environment ${ }^{3}$. An organism that exists only in a mycelial form in the soil is unlikely to be identified from direct observation if a fruiting body is not formed. Therefore, classical observation through direct microscopy will give a greatly reduced measure of the true diversity in the environment. Culturing fungi from soil isolations will only result in the detection of those propagules that are able to grow and sporulate on the isolation medium used. Nanotechnology is emerging field of science which involves synthesis and development of various nanomaterials. Silver nano particles have found potential application in many fields such as, antibacterial effect, biological sensors, drug delivery, textile and filters. Nanoparticles can be synthesized by physical, chemical and biological methods. Fungi are ideal candidates in the synthesis of metal nanoparticles, because of their ability to secrete large amount of enzymes. The objective of our study is to find the biodiversity of fungi associated with Silver ore soil collected from Chennai, Tamil Nadu, India and to study the presence of silver nanoparticles production from any species ${ }^{4-8}$.

\section{MATERIALS AND METHODS}

The soil samples were collected every month from May to October 2012. The soil dilution technique and direct plating technique was used to determine the number of fungal colonies in soil samples. To isolate pure cultures, Plates with PDA medium supplemented with chloramphenicol was used. The inoculated petri dishes were incubated in a sterile culture room at $25^{\circ} \mathrm{C} \pm 1{ }^{\circ} \mathrm{C}$. Colony forming units (CFU) were estimated by counting the number of colonies after five days. Fungal colonies formed were calculated on per gram dry soil basis.

\section{Isolation of Fungi}

Fungi were identified based on their macroscopic and microscopic features. Identification at the species level was carried out according to the morphological characters found principally in literatures. Pure cultures of fungi were maintained in test tubes slants containing Potato Dextrose agar medium and preserved in deep freezer at $20^{\circ} \mathrm{C}^{9-15}$.

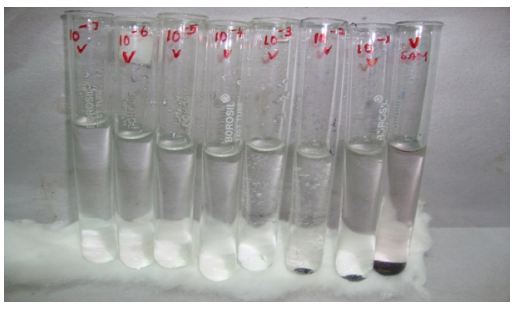

Figure 2: Serial Dilution of the Soil Sample 


\section{RESULT AND DISCUSSION}

During the study the soil samples were processed and the fungi were identified. Of these most of the fungi belonged to Aspergillus species. The statistical analysis was made to find out which was the most grown fungi from the serial dilution technique, during the period of study. Further the fungal morphological characters were studied in detail for the silver nanoparticle production. The list of identified fungi are as follows.

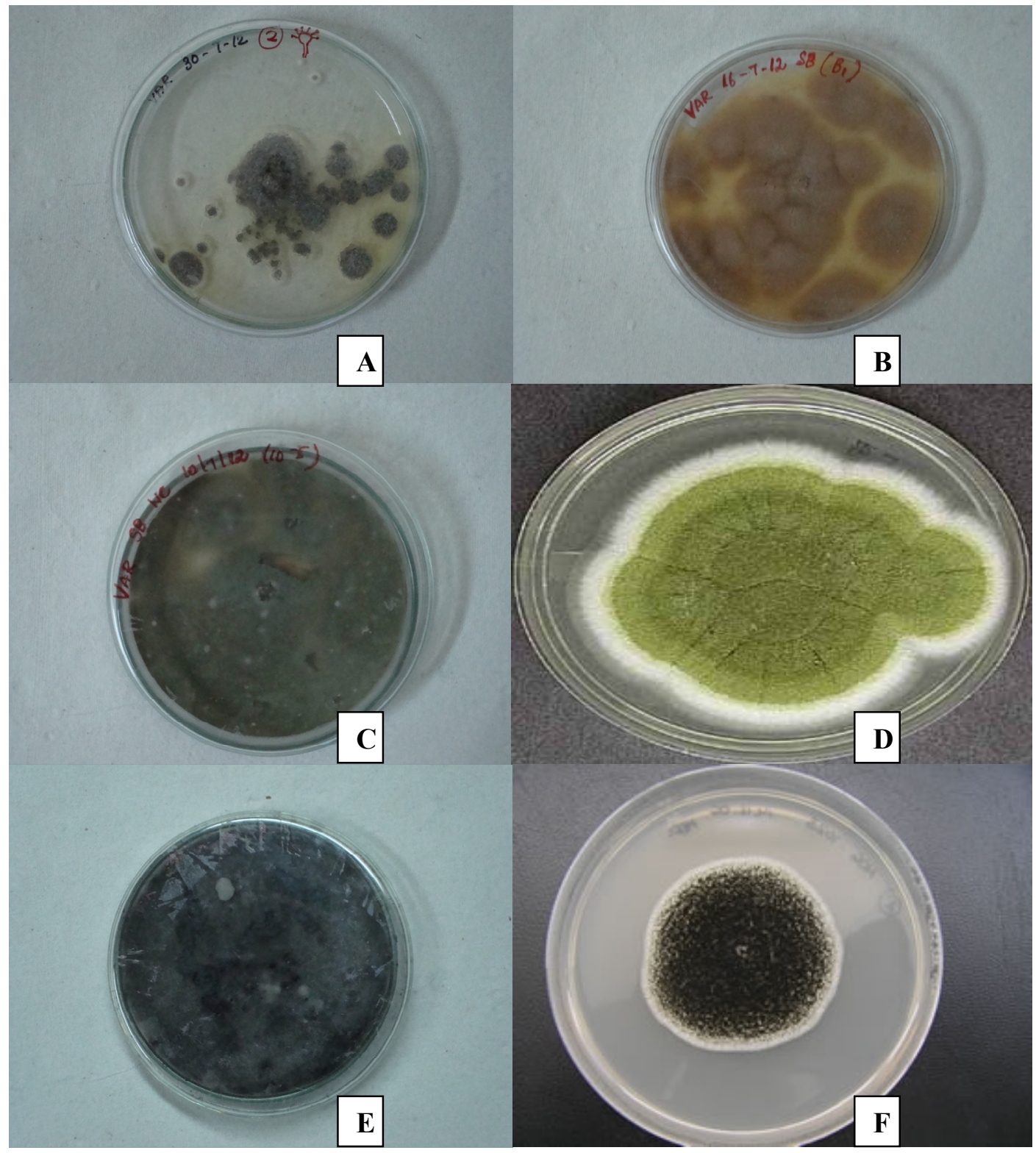

Figure 1: Fungal cultures isolated from silver ore soil sample

A) Aspergillus japonicus, B) Aspergillus tereus, C) Aspergillus nidulans, D) Aspergillus flavus, E) Bortyodiplodia F) Aspergillus niger

Of the grown fungi, it is found that the most abundantly grown species was Aspergillus niger. Taking the frequency of the growth of this fungus, it is considered for the analysis of silver nanoparticle synthesis.

\section{Synthesis of Silver Nanoparticles}

The selected fungi were repeatedly cultured for purity and the culture was maintained in PDA slants for future use. As the fungi were isolated from silver ore, it was mass cultured $1 \mathrm{~L}$ Erlenmeyer flask containing PDB medium amended with chloramphenicol. The flask was inoculated for 3-4 weeks and the fungal mat was harvested by passing through a four layered cheese cloth. The harvested mat was fixed using gluteraldehyde.

\section{Scanning Electron Microscopy (SEM)}

The Fungi were allowed to dry completely by fixing the fungal mat at various percentage of acetone. Finally the fungal samples were fixed in $100 \%$ acetone for SEM analysis. Since the specimen is at high vacuum, Living cells and tissues and whole, soft-bodied organisms usually require chemical fixation to preserve and stabilize. Fixation is usually performed by incubation in a solution of a buffered chemical fixative, such as glutraldehyde. The fixed tissue is then dehydrated. 


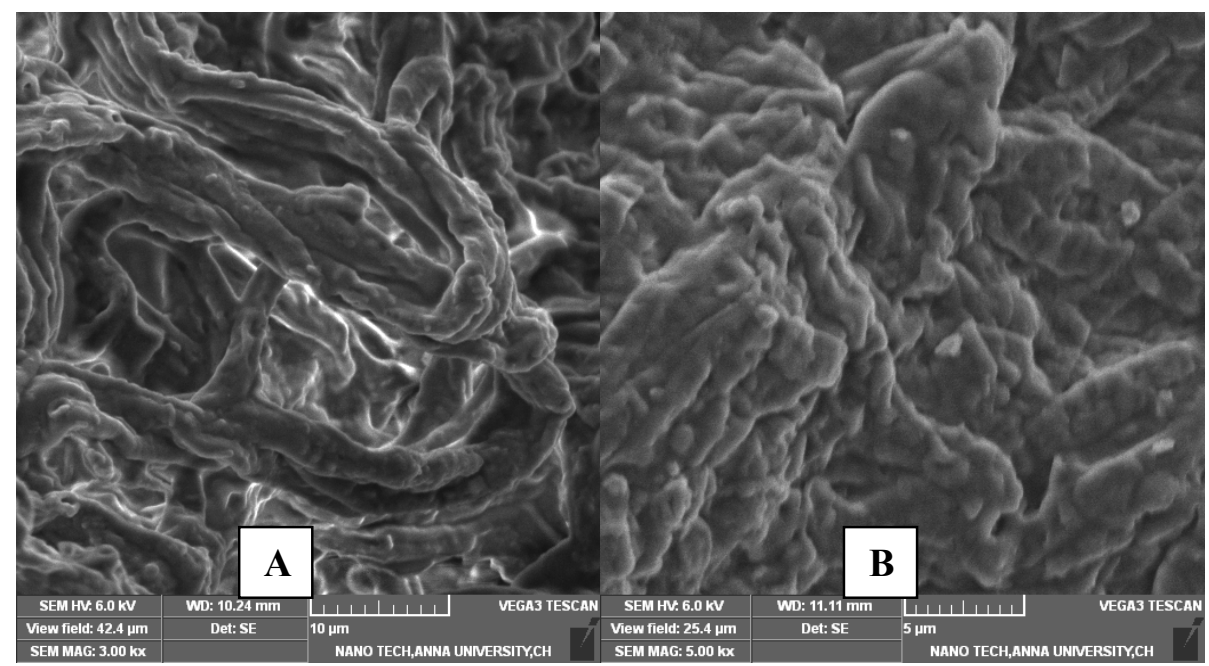

Figure 2: (A) Control (B) SEM analysis of Aspergillus niger isolated from silver ore soil, showing different morphology in fungal mycelia indicates the synthesis of silver nanoparticles

\section{CONCLUSION}

As an important component of soil micro organism, fungi take a very important position in structure and function of ecosystem. Fungi therefore have many different functions in soils, which includes both active roles, such as the degradation of dead plant material, or inactive roles where propagules are present in the soil as resting states. From the above study it is concluded that hypomycetes are the largest group of fungi that is found in the silver ore soil sample collected. Of the Fungi isolated, Aspergillus niger is used for the analysis of silver nanoparticle production. It is found that the fungi are capable of producing silver nanoparticle. The produced nanoparticle can be further used in the cell lines to find out various activities of the nanoparticle being produced such as cytotoxicity and cancer studies.

\section{ACKNOWLEDGEMENTS}

The authors express sincere thanks to INSPIRE, Department of Science and Technology (DST), India, for their valuable support and providing necessary funds to carry out the research work. The authors also thank CNST, Anna University, Chennai, Tamil Nadu, India for the instrumentation facility.

\section{REFERENCES}

1. JH Warcup. The Soil Plate Method for Isolation of Fungi from Soil. Nature 166; 1950. p. 117-118. http://dx.doi.org/10.1038/ $166117 \mathrm{~b} 0$

2. D Parkinson and ST Williams. A method for isolating fungi from soil microhabitats. Plant and soil Journal 1960; 13(4): 347-355. http://dx.doi.org/10.1007/BF01394646

3. Thomas Pumpel and Franz Schinner. Silver tolerance and silver accumulation of microorganisms from soil materials of silver mine. Applied microbiology and Biotechnology 1986; 24(3): 244-247. http://dx.doi.org/10.1007/BF00261545

4. Shaheen Zafar, Farrukh Aqil and Iqbal Ahmad. Metal tolerance and bio sorption potential of filamentous fungi isolated from metal contaminated agricultural soil. Bio resource technology 2007; 98(13): 2557-2561. http://dx.doi.org/10.1016 j.biortech.2006.09.051

5. Saeed Moharrer, Behroz Mohammadi, Reza Azizi Gharamohammadi and Mehdi Yargoli. Biological synthesis of silver nanoparticles by Aspergillus flavus, isolated from soil of Ahar copper mine. Indian Journal of Science and Technology 2012; 5(S3): 2443-2444.
6. Zhong WH, Cai ZC. Methods for studying soil microbial diversity. Chinese Journal of Applied Ecology 2004; 15(5): 899904.

7. Ogram A. Soil molecular microbial ecology at age 20: methodological challenges for the future. Soil Biology and Biochemistry 2000; 32(11-12): 1499-1504. http://dx.doi.org/ 10.1016/S0038-0717(00)00088-2

8. Castro Longoria E, Vilchis Nestor AR and Avalos Borja M. Biosynthesis of silver, gold and bimetallic nanoparticles using the filamentous fungus Neurospora crassa. Colloids Surf. B Bio interfaces 2010; 23: 112-117.

9. Nameirakpam Nirjanta Devi, Dheeban Shankar P and Sutha S. Bio mimetic synthesis of silver nanoparticles from an endophytic fungus and their antimicrobial efficacy. International Journal of Biomedical and Advance Research 2012; 03(05).

10. Hemath Naveen KS, Gaurav Kumar, Karthik L and Bhaskara Rao KV. Extracellular biosynthesis of silver nanoparticles using the filamentous fungus Penicillium sp. Scholars Research Library Archives of Applied Science Research 2010; 2(6): 161167.

11. Gajendran N. Adding Life to the nanotechnology. Indian J. Sci. Technol 2009; 1(1): 1-5.

12. Kathiresan K, Manivannan S, Nabeel MA and Dhivya B. Studies on silver nanoparticles synthesized by a marine fungus, Penicillium fellutanum isolated from coastal mangrove sediment. Colloids Surf. B Bio interfaces 2009; 71: 133-137. http://dx.doi.org/10.1016/j.colsurfb.2009.01.016

13. Narayanan KB and Sakthivel N. Biological synthesis of metal nanoparticles by microbes. Adv. Colloid Interface Sci 2010; 156: 1-13. http://dx.doi.org/10.1016/j.cis.2010.02.001

14. Lurdes Barrico, Susana Rodríguez Echeverría and Helena Freitas. Diversity of soil basidiomycete communities associated with Quercus suber L. in Portuguese montados European Journal of Soil Biology 2010; 1-8.

15. Karin Vega, Gretty K Villena, Victor H Sarmiento, Yvette Ludena, Nadia Vera and Marcel Gutierrez Correa. Production of Alkaline Cellulase by Fungi Isolated from an Undisturbed Rain Forest of Peru. Biotechnology Research International; 2012. http://dx.doi.org/10.1155/2012/934325

\section{Cite this article as:}

Janakiraman Vardhana, Govindarajan Kathiravan, Ravichandran Dhivya, Doss Vidhya. Biodiversity of fungi from silver ore with reference to the production of silver nanoparticles. Int. Res. J. Pharm. 2015; 6(1):31-33 http://dx.doi.org/10.7897/2230-8407.0617 